\title{
Terminal sliding mode control for continuous stirred tank reactor
}

\author{
Dongya Zhao ${ }^{1, *}$, Quanmin Zhu ${ }^{1,2}$, Johan Dubbeldam ${ }^{3}$ \\ 1 College of Chemical Engineering, China University of Petroleum, Qingdao, P. R. China, 266580 \\ 2 Faculty of Environment and Technology, University of the West of England, Coldharbour Lane, Bristol BS16 1QY, UK \\ 3 Department of Applied Mathematics, Delft University of Technology, Mekelweg 4, 2628CD, Delft, Netherlands \\ * Corresponding Author: dongyazhao@gmail.com; dyzhao@upc.edu.cn
}

\author{
Abbreviation index \\ CSTR--continuous stirred tank reactor \\ FITSMC--fraction integral terminal sliding mode control \\ I/O--input/output \\ ISMC--integral sliding mode control \\ ITSE--integral of time multiplied by squared error \\ SITSMC --sign integral terminal sliding mode control \\ SM--sliding mode \\ TSM--terminal sliding mode \\ TSMC--terminal sliding mode control
}

Table caption index

Table 1 Dimensionless parameters for the CSTR model

Table 2 The controllers' parameters

Table 3 Integral of time multiplied by squared error (ITSE)

\section{Figure caption index}

Figure 1 CSTR diagrammatic sketch 
Figure 2 (a) $\hat{x}_{1}$ and $x_{1}$ of SITSMC (Non-smooth)

Figure 2 (b) $\hat{x}_{2}$ and $x_{2}$ of SITSMC (Non-smooth)

Figure 2 (c) Control input of SITSMC (Non-smooth)

Figure 3 (a) $\hat{x}_{1}$ and $x_{1}$ of FITSMC (Non-smooth)

Figure 3 (b) $\hat{x}_{2}$ and $x_{2}$ of FITSMC (Non-smooth)

Figure 3 (c) Control input of FITSMC (Non-smooth)

Figure 4 (a) $\hat{x}_{1}$ and $x_{1}$ of SITSMC (Smooth)

Figure 4 (b) $\hat{x}_{2}$ and $x_{2}$ of SITSMC (Smooth)

Figure 4 (c) Control input of SITSMC (Smooth)

Figure 5 (a) $\hat{x}_{1}$ and $x_{1}$ of FITSMC (Smooth)

Figure 5 (b) $\hat{x}_{2}$ and $x_{2}$ of FITSMC (Smooth)

Figure 5 (c) Control input of FITSMC (Smooth)

Figure 6 (a) $\hat{x}_{1}$ and $x_{1}$ of ISMC (Smooth)

Figure 6 (b) $\hat{x}_{2}$ and $x_{2}$ of ISMC (Smooth)

Figure 6 (c) Control input of ISMC (Smooth)

Figure 7 (a) $\hat{x}_{2}$ and $x_{2}$ of SITSMC under external disturbances (Smooth)

Figure 7 (b) $\hat{x}_{2}$ and $x_{2}$ of FITSMC under external disturbances (Smooth)

Figure 7 (c) $\hat{x}_{2}$ and $x_{2}$ of ISMC under external

\section{Terminal sliding mode control for continuous stirred tank reactor}

Dongya Zhao ${ }^{1, *}$, Quanmin Zhu ${ }^{1,2}$, Johan Dubbeldam ${ }^{3}$

1 College of Chemical Engineering, China University of Petroleum, Qingdao, P. R. China, 266580

2 Faculty of Environment and Technology, University of the West of England, Coldharbour Lane, Bristol BS16 1QY, UK

3 Department of Applied Mathematics, Delft University of Technology, Mekelweg 4, 2628CD, Delft, Netherlands

* Corresponding Author: dongyazhao@gmail.com; dyzhao@upc.edu.cn 


\begin{abstract}
A continuous stirred tank reactor (CSTR) is a typical example of chemical industrial equipment, whose dynamics represent an extensive class of second order nonlinear systems. It has been witnessed that designing a good control algorithm for the CSTR is very challenging due to the high complexity. The two difficult issues in CSTR control are state estimation and external disturbance attenuation. In general, in industrial process control a fast and robust response is essential. Driven by these challenging issues and desired performance, this paper proposes an output feedback terminal sliding mode control (TSMC) framework which is developed for CSTR, and can estimate the system states and stabilize the system output tracking error to zero in a finite time. The corresponding stability analysis is presented in terms of the Lyapunov method. Illustrative examples are demonstrated by using Matlab simulations to validate the effectiveness of the proposed approach.
\end{abstract}

\title{
Keywords:
}

CSTR; State observer; Terminal sliding mode control; Finite time stability

\section{Introduction}

CSTR is one of the most common used equipment in the process industries. It can convert reactants into products, and therefore plays a primary role in many chemical processes ${ }^{1-4}$. In general, CSTRs are operated around a certain equilibrium point linked to the optimal output or optimal productivity of a process to pursue a high conversion rate and maximize economic benefits. In the view of control, CSTRs are highly nonlinear and dynamic. They have some notable features, such as one relative degree, unmeasured states and zero dynamics. These features make the controller design very challenging, especially in the presence of the external disturbance and/or system uncertainty ${ }^{5-8}$. In a wider sense, the investigations on the control solutions for the CSTRs can be extended to other processes by slight modifications.

There has been much effort in the design of controlling CSTRs. By using Taylor-linearization for the dynamic models with bounded uncertainty, linear controllers have been presented ${ }^{9-10}$. However, the global stability may be lost while using the local linear approximation ${ }^{11}$. In the light of full state feedback and coordinate transformation, robust control approaches have been developed to achieve the disturbance attenuation performance ${ }^{12-13}$. Note that it is very difficult to measure the concentration of the reactant directly online in practice. Hence full state feedback control is not practical in applications. However, it has been found that input/output (I/O) feedback linearization is 
a practical control approach. Nevertheless, it still requires knowledge of the unmeasured states by the I/O feedback $^{7}$. To resolve this issue, some observer based nonlinear control approaches have been presented ${ }^{14-20}$. Some advanced industrial applications, e.g. alkylation of benzene with ethylene process, expect high performance such as, strong robustness and fast response. Even the above mentioned observer based control approaches can in practice only achieve (asymptotical) stability, when high gain control is applied. Such high gain control may lead to control input saturation, particularly in the instance of the large initial track error ${ }^{21-22}$. If there exist external disturbances, it is very challenging to design observers and output feedback controllers for CSTRs.

A nonlinear sliding mode (SM) control approach named terminal sliding mode (TSM) has been proposed for the nonlinear system control. It has some important advantages such as fast converging speed, strong robustness to system uncertainty, external disturbance and finite time stability ${ }^{23-29}$ without requiring high control gains. Recently, such superior control method has been successfully used in industrial processes ${ }^{30}$, which provides a good illustration of how TSMC can be utilized in process industry. Note that, the TSMC approach was developed in [30] for a plate heat exchanger ${ }^{30}$, which can be linearized by state feedback. Due to the properties such as relative degree one, unmeasured states and zero dynamics of the CSTRs, the method developed in [30] cannot be applied to CSTR directly. Note that sliding mode observers have strong ability to estimate system states, external disturbances, whose principle will be used to design a finite time stability observer in this paper. The sliding state observer is strongly robust and fast converging which is a good choice for observer based controller design ${ }^{31-32}$. Note that most of the existing sliding mode observers are asymptotically stable which cannot be used in the finite time stability control.

The purpose of this study is to design a novel output feedback TSMC for a class of CSTRs, which have stable zero dynamics. In practical situations, external disturbances will be presented and affect the CSTR dynamics, which enhances the difficulties of controller design. First, a finite time stability state observer is proposed to estimate the unmeasured states online. Then, a TSMC is developed for CSTRs. The novelty of this paper is that it focuses on the finite time stability of the temperature loop in the presence of an external disturbance in the concentration loop. Compared with the existing sliding mode control approaches for $\operatorname{CSTR}^{7,33-34}$, the proposed approach has a much stronger robustness and faster converging speed without requiring high control gain.

The rest of this paper is organized as follows: The dynamic equations of a CSTR and some indispensable preliminary knowledge are presented in Section 2. Output feedback TSMC and the corresponding stability analysis are given in Section 3. Illustrative examples are used to validate the effectiveness of the proposed approaches in Section 4. Finally, concluding remarks are given in Section 5. 


\section{Problem formulation and preliminaries}

\subsection{Dynamic equation of a CSTR}

Here we consider a CSTR as an exothermic, first order, irreversible reaction with the following two assumptions

Assumption 1: The temperature is uniformly distributed due to perfect mixing in the reactor. The reacting materials have constant density and capacity.

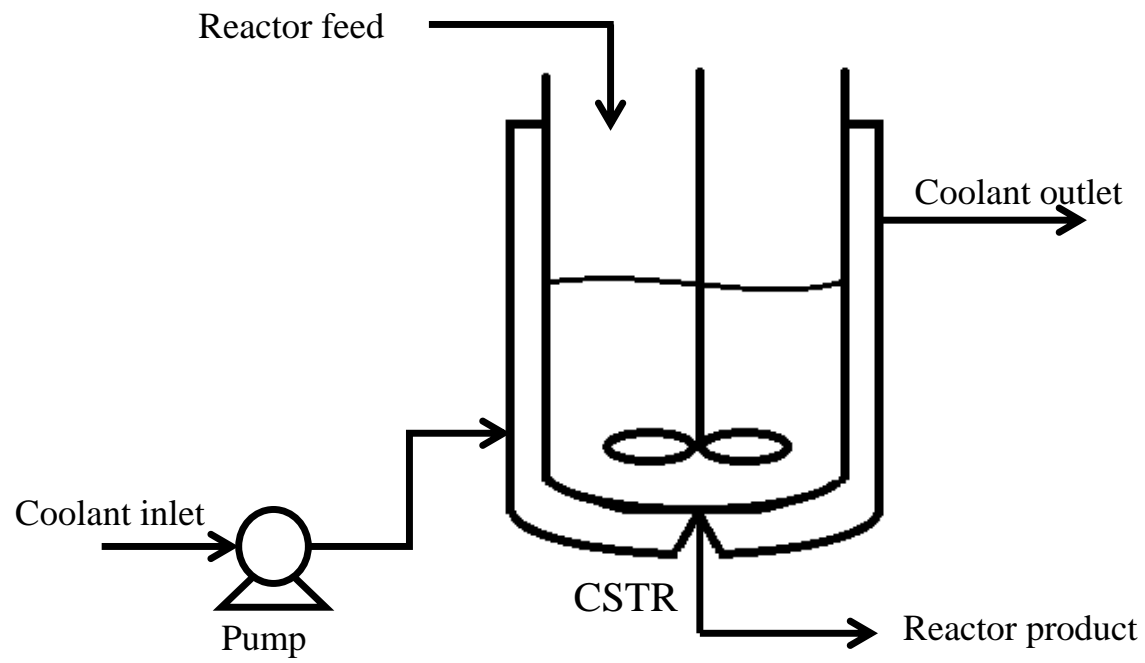

Figure 1 CSTR diagrammatic sketch

Therefore, a dimensionless dynamic equation of the CSTR (as shown in Figure 1) can be used to describe the exothermic, first order, irreversible reaction ${ }^{7}$ :

$$
\begin{aligned}
& \dot{x}_{1}=-x_{1}+D_{a}\left(1-x_{1}\right) e^{x_{2} /\left(x_{2} / \gamma+1\right)}-d_{1} \\
& \dot{x}_{2}=-x_{2}+B D_{a}\left(1-x_{1}\right) e^{x_{2} /\left(x_{2} / \gamma+1\right)}-\beta\left(x_{2}-x_{2 c}\right)+\beta u+d_{2} \\
& y=x_{2}
\end{aligned}
$$

where $x_{1}, x_{2} \in R$ are the states, $y$ is the system output which represents the dimensionless temperature, $d_{1}, d_{2} \in R$ are external disturbances in the inlet concentration and temperature respectively. The details of the dimensionless parameters of this dynamic equation are given in Table 1 with references to the literature 7.

Remark 1: For interested control, dimensionless temperature is selected as the system output for the following two reasons (1) Concentration measurement is very expensive in general; (2) To avoid secondary reactions, the reactor temperature has a maximum restriction ${ }^{7}$. 
Property 1: The dynamic equation (1) has a relative degree one.

Property 2: $\dot{x}_{1}=-x_{1}+D_{a}\left(1-x_{1}\right) e^{x_{2} /\left(x_{2} / \gamma+1\right)}-d_{1}$ is zero dynamics.

Assumption 2: $\left|d_{1}\right| \leq c_{1}, c_{1}>0 . d_{2}$ is a measurable disturbance that is bounded.

Assumption 3: The control input $u(t)$ belongs to the extended $L_{p}$ space denoted as $\tilde{L}_{p}$. That is, any truncation of $u(t)$ to a finite time interval is essentially bounded ${ }^{35}$.

Remark 2: Assumptions 2 and 3 are realistic. Because $d_{2}$ denotes a feed temperature disturbance it can be measured by using thermocouples. A properly designed control algorithm must lead to a bounded controller output, otherwise saturation or limit effects will degrade the control performance, and may even cause instability.

Remark 3: According to Properties 1-2 and Assumptions 2 and 3, the zero dynamics of (1) must be stable. An output feedback controller can guarantee the whole system to be stable.

The control objective of this paper is summarized as: Design a finite time stable observer to estimate system states. Then develop an output feedback terminal sliding mode control algorithm with the estimated states. It can drive the system output to its desired operation point while guaranteeing the concentration to be stable.

Table 1 Dimensionless parameters for the CSTR model

\begin{tabular}{|l|l|}
\hline Activation energy & $\gamma=E / R T_{0}$ \\
\hline Adiabatic temperature rise & $B=(-\Delta H) c_{A f_{0}} / \rho c_{p} T_{f_{0}}$ \\
\hline Damkohler number & $D_{a}=k_{0} \exp (-\gamma V) / V$ \\
\hline Heat transfer coefficient & $\beta=h A / \rho c_{p} f_{0}$ \\
\hline Dimensionless time & $t=t^{\prime}\left(F_{0} / V\right)$ \\
\hline Dimensionless composition & $x_{1}=\left(c_{A f_{0}}-c_{A}\right) / c_{A f_{0}}$ \\
\hline Dimensionless temperature & $x_{2}=\left(T-T_{f_{0}}\right) / T_{f_{0}}$ \\
\hline Dimensionless control input & $u=\left(T_{c}-T_{c_{0}}\right) / T_{f_{0}}$ \\
\hline Feed composition disturbance & $d_{1}=\left(c_{A f}-c_{A f_{0}}\right) / c_{A f_{0}}$ \\
\hline Feed temperature disturbance & $d_{2}=\left(T_{f}-T_{f_{0}}\right) / T_{f_{0}}$ \\
\hline
\end{tabular}

\subsection{Integral terminal sliding mode control}

Consider a nonlinear system as follows:

$$
\dot{x}=f(x)+g(x) u,
$$


where $x \in R, f(x) \in R, g(x) \in R$ and $g^{-1}(x) \in R$ exists and is bounded.

Two integral TSM were presented in Ref. [23]:

A. Sign integral terminal sliding mode

$$
\begin{aligned}
& s(t)=x(t)+\lambda x_{I}(t) \\
& \dot{x}_{I}(t)=\operatorname{sgn}(x(t)) \quad x_{I}(0)=-x(0) / \lambda
\end{aligned}
$$

where $\lambda>0, x(0)$ is the initial value of $x(t)$. According to the principle of sliding mode control ${ }^{36}, x(t)$ will be always kept on $s(t)$. If $s(t)$ is always zero, $x(t)$ will converge to zero in a finite time $T_{s}=|e(0)| / \alpha$.

\section{B. Fraction integral terminal sliding mode}

$$
\begin{aligned}
& s(t)=x(t)+\lambda x_{I}(t) \\
& \dot{x}_{I}(t)=|x(t)|^{q / p} \operatorname{sgn}(x(t)) \quad x_{I}(0)=-x(0) / \lambda
\end{aligned}
$$

where $p, q>0$ are odd integers. In light of the definition of a $\mathrm{TSM}^{23}, x(t)$ will be zero in a finite time $T_{s}=|e(0)|^{1-q / p} / \alpha(1-q / p)$

By using (3), TSMC can be designed for (2):

$$
u=g^{-1}(x)(-f(x)-\alpha \operatorname{sgn}(x)-\eta \operatorname{sgn}(s))
$$

By using (4), TSMC can be designed for (2):

$$
u=g^{-1}(x)\left(-f(x)-\alpha|x|^{q / p} \operatorname{sgn}(x)-\eta \operatorname{sgn}(s)\right)
$$

Control laws (5) and (6) can force $x(t)$ to remain in the TSM, consequently it will converge to zero in finite time along TSM.

\section{Output feedback TSMC for CSTR}

Because $x_{1}$ cannot be measured, full state feedback control is not feasible for (1). Though there have been designed many observers for CSTRs by means of asymptotical stability or practical stability, if used in TSMC, finite time stability will be lost. Accordingly, a finite time stable observer should be designed first. 


\subsection{Finite time stability observer}

Design the following finite time stability observer:

$$
\begin{aligned}
& \dot{\hat{x}}_{1}=-\hat{x}_{1}+D_{a}\left(1-\hat{x}_{1}\right) e^{\hat{x}_{2} /\left(\hat{x}_{2} / \gamma+1\right)}+\alpha_{1} \operatorname{sgn}\left(\bar{x}_{1}-\hat{x}_{1}\right) \\
& \dot{\hat{x}}_{2}=-\hat{x}_{2}+B D_{a}\left(1-\hat{x}_{1}\right) e^{\hat{x}_{2} /\left(\hat{x}_{2} / \gamma+1\right)}-\beta\left(\hat{x}_{2}-x_{2 c}\right)+\beta u+d_{2}+\alpha_{2} \operatorname{sgn}\left(y-\hat{x}_{2}\right)
\end{aligned}
$$

where $\hat{x}_{1}, \hat{x}_{2} \in R$ are estimated states for $x_{1}$ and $x_{2}, \alpha_{1}, \alpha_{2}>0$ are positive numbers. Let $M=e^{x_{2} /\left(x_{2} / \gamma+1\right)}$ and $\hat{M}=e^{\hat{x}_{2} /\left(\hat{x}_{2} / \gamma+1\right)}$, then $\bar{x}_{1}$ is defined as:

$$
\bar{x}_{1}=\hat{x}_{1}-\left(\alpha_{2} \operatorname{sgn}\left(y-\hat{x}_{2}\right)\right)_{e q} / B D_{a} \hat{M}
$$

Define the estimating error as:

$$
\begin{aligned}
& \tilde{x}_{1}=x_{1}-\hat{x}_{1} \\
& \tilde{x}_{2}=x_{2}-\hat{x}_{2}
\end{aligned}
$$

According to (9):

$$
\begin{aligned}
& \dot{\tilde{x}}_{1}=-\tilde{x}_{1}+D_{a} M\left(1-x_{1}\right)-D_{a} \hat{M}\left(1-\hat{x}_{1}\right)-d_{1}-\alpha_{1} \operatorname{sgn}\left(\bar{x}_{1}-\hat{x}_{1}\right) \\
& \dot{\tilde{x}}_{2}=-(1+\beta) \tilde{x}_{2}+B D_{a} M\left(1-x_{1}\right)-B D_{a} \hat{M}\left(1-\hat{x}_{1}\right)-\alpha_{2} \operatorname{sgn}\left(y-\hat{x}_{2}\right)
\end{aligned}
$$

where $\left(\alpha_{2} \operatorname{sgn}\left(y-\hat{x}_{2}\right)\right)_{e q}$ is the equivalent output injection of $\alpha_{2} \operatorname{sgn}\left(y-\hat{x}_{2}\right)$. It can be obtained by passing the signal through a low pass filter ${ }^{37}$.

Theorem 1: Under Assumptions 1-3, observer (7) can be used to estimate system states $x_{1}$ and $x_{2}$ in a finite time, that is, $\tilde{x}_{1}$ and $\tilde{x}_{2}$ tend to zero in a finite time.

\section{Proof:}

Choose a Lyapunov function for the temperature estimating loop as follows:

$$
V_{2}=\frac{1}{2} \tilde{x}_{2}^{2}
$$

By differentiating (11) with respect to time along the temperature estimating loop yields:

$$
\begin{aligned}
\dot{V}_{2} & =\tilde{x}_{2} \dot{\tilde{x}}_{2}=\tilde{x}_{2}\left[-(1+\beta) \tilde{x}_{2}+B D_{a} M\left(1-x_{1}\right) \tilde{x}_{2}-B D_{a} \hat{M}\left(1-\hat{x}_{1}\right)\right]-\alpha_{2}\left|\tilde{x}_{2}\right| \\
& \leq-\alpha_{2}\left|\tilde{x}_{2}\right|+\left|\tilde{x}_{2}\right|\left|-(1+\beta) \tilde{x}_{2}+B D_{a} M\left(1-x_{1}\right) \tilde{x}_{2}-B D_{a} \hat{M}\left(1-\hat{x}_{1}\right)\right| \\
& =-\left|\tilde{x}_{2}\right|\left(\alpha_{2}-\left|-(1+\beta) \tilde{x}_{2}+B D_{a} M\left(1-x_{1}\right) \tilde{x}_{2}-B D_{a} \hat{M}\left(1-\hat{x}_{1}\right)\right|\right)
\end{aligned}
$$

If we choose $\alpha_{2}$ large enough, that is $\alpha_{2} \geq\left|-(1+\beta) \tilde{x}_{2}+B D_{a} M\left(1-x_{1}\right) \tilde{x}_{2}-B D_{a} \hat{M}\left(1-\hat{x}_{1}\right)\right|, \quad \tilde{x}_{2}$ and $\dot{\tilde{x}}_{2}$ will converge to zero in finite time ${ }^{36}$. Note that, as $\tilde{x}_{2}=0$ and $\dot{\tilde{x}}_{2}=0,-B D_{a} \hat{M} \tilde{x}_{1}=\alpha_{2}\left(\operatorname{sgn}\left(y-\hat{x}_{2}\right)\right)_{e q}$. 
By using a low pass filter ${ }^{37}$, one can obtain the equivalent output of $\alpha_{2} \operatorname{sgn}\left(y-\hat{x}_{2}\right)$.

Consider $\tilde{x}_{2}=0$ in finite time, (8) can be written as:

$$
\dot{\tilde{x}}_{1}=-\tilde{x}_{1}-D_{a} \hat{M} \tilde{x}_{1}-d_{1}-\alpha_{1} \operatorname{sgn}\left(\bar{x}_{1}-\hat{x}_{1}\right)
$$

If we choose a Lyapunov function for concentration estimating loop as follows:

$$
V_{1}=\frac{1}{2} \tilde{x}_{1}^{2}
$$

By differentiating (14) with respect to time along the concentration estimating loop, it will be:

$$
\begin{aligned}
\dot{V}_{1} & =\tilde{x}_{1} \dot{\tilde{x}}_{1} \\
& =\tilde{x}_{1}\left(-\tilde{x}_{1}+D_{a} M\left(1-x_{1}\right)-D_{a} \hat{M}\left(1-\hat{x}_{1}\right)-d_{1}-\alpha_{1} \operatorname{sgn}\left(\bar{x}_{1}-\hat{x}_{1}\right)\right) \\
& =-\alpha_{1}\left|\tilde{x}_{1}\right|-\tilde{x}_{1}\left(\tilde{x}_{1}+D_{a} \hat{M} \tilde{x}_{1}+d_{1}\right) \\
& \leq-\alpha_{1}\left|\tilde{x}_{1}\right|+\left|\tilde{x}_{1}\right|\left|\tilde{x}_{1}+D_{a} \hat{M} \tilde{x}_{1}+d_{1}\right| \\
& =-\left|\tilde{x}_{1}\right|\left(\alpha_{1}-\left|\tilde{x}_{1}+D_{a} \hat{M} \tilde{x}_{1}+d_{1}\right|\right)
\end{aligned}
$$

If one chooses $\alpha_{1}$ is large enough, $\alpha_{1}-\left|\tilde{x}_{1}+D_{a} \hat{M} \tilde{x}_{1}+d_{1}\right|>0, \quad \tilde{x}_{1}$ and $\dot{\tilde{x}}_{1}$ will converge to zero in finite time $^{36}$.

\subsection{Output feedback TSMC design for CSTR}

Suppose the desired trajectory of system output as:

$$
\begin{aligned}
& y_{r}=x_{2 s}\left(1-k_{1} e^{-k_{2} t}\right) \\
& \dot{y}_{r}=k_{1} k_{2} x_{2 s} e^{-k_{2} t}
\end{aligned}
$$

where $k_{1}, k_{2}>0$ are positive constants that depend on the practical restrictions. $x_{2 s}$ is the desired steady state value of $x_{2}$, which should be bounded.

Define the tracking error of system output as:

$$
e=x_{2}-y_{r}
$$

The estimated tracking error should be:

$$
\begin{aligned}
& \hat{e}=\hat{x}_{2}-y_{r} \\
& \dot{\hat{e}}=-\hat{x}_{2}+B D_{a}\left(1-\hat{x}_{1}\right) \hat{M}-\beta\left(\hat{x}_{2}-x_{2 c}\right)+\beta u+d_{2}+\alpha_{2} \operatorname{sgn}\left(y-\hat{x}_{2}\right)-\dot{y}_{r}
\end{aligned}
$$

\section{A. Sign integral terminal sliding mode control}

For (18), the estimated sign integral terminal sliding mode is: 


$$
\begin{aligned}
& \hat{s}=\hat{e}+\lambda \hat{e}_{I} \\
& \dot{\hat{e}}_{I}=\operatorname{sgn}(\hat{e}) \quad \hat{e}_{I}(0)=-\hat{e}(0) / \lambda
\end{aligned}
$$

The terminal sliding mode can be re-written as:

$$
\hat{s}=\hat{e}+\lambda \int_{0}^{t} \operatorname{sgn}(\hat{e}(\tau)) d \tau
$$

If $\hat{e}$ reaches $\hat{s}$ it will converge to zero in a finite time $T_{s}=|e(0)| / \lambda$.

The derivative of $\hat{s}$ is:

$$
\dot{\hat{s}}=\dot{\hat{e}}+\lambda \operatorname{sgn}(\hat{e})
$$

Let $\dot{\hat{S}}=0$, one can get the equivalent control as:

$$
\begin{aligned}
u_{e q} & =\beta^{-1}\left(\hat{x}_{2}-B D_{a}\left(1-\hat{x}_{1}\right) e^{\hat{x}_{2} /\left(\hat{x}_{2} / \gamma+1\right)}+\beta\left(\hat{x}_{2}-x_{2 c}\right)\right. \\
& \left.-d_{2}-\alpha_{2} \operatorname{sgn}\left(y-\hat{x}_{2}\right)+\dot{y}_{r}-\lambda \operatorname{sgn}(\hat{e})\right)
\end{aligned}
$$

Design the reaching law as:

$$
u_{s}=-K \operatorname{sgn}(\hat{s})
$$

where $K>0$ is a positive number.

The terminal sliding mode controller is designed as:

$$
u=u_{e q}+u_{s}
$$

Theorem 2: Under Assumptions 1-3 and Properties 1-2, the sign integral TSMC (24) yields convergence of $\hat{e}$ and $\dot{\hat{e}}$ to 0 in finite time, and hence $x_{2}$ will track to $y_{r}$ in finite time and $x_{1}$ is asymptotically stable.

\section{Proof:}

Choose Lyapunov function as:

$$
V_{1}=\frac{1}{2} \hat{s}^{2}
$$

Differentiate (25) with respect to time along (18):

$$
\begin{aligned}
\dot{V}_{1} & =\hat{s} \dot{s} \\
& =\hat{s}\left(-\hat{x}_{2}+B D_{a}\left(1-\hat{x}_{1}\right) \hat{M}-\beta\left(\hat{x}_{2}-x_{2 c}\right)\right. \\
& \left.+\beta u+d_{2}+\alpha_{2} \operatorname{sgn}\left(y-\hat{x}_{2}\right)-\dot{y}_{r}+\operatorname{sgn}(\hat{e})\right)
\end{aligned}
$$

Substituting (24) into (26):

$$
\dot{V}_{1}=-K|\hat{s}|
$$


According to the sliding condition ${ }^{36}, \hat{e}$ is kept on TSM (20) all the time. From Theorem 1, it converges to zero in finite time.

Note that after the after $\hat{x}_{1}$ and $\hat{x}_{2}$ converge to $x_{1}$ and $x_{2}$, the control law should be:

$$
\begin{aligned}
\bar{u}_{e q} & =\left(x_{2}-B D_{a}\left(1-x_{1}\right) e^{x_{2} /\left(x_{2} / \gamma+1\right)}+\beta\left(x_{2}-x_{2 c}\right)-d_{2}\right. \\
& \left.-\alpha_{2} \operatorname{sgn}\left(y-x_{2}\right)+\dot{y}_{r}-\lambda \operatorname{sgn}(e)\right)
\end{aligned}
$$

The reaching law is

$$
\bar{u}_{s}=-K \operatorname{sgn}(s)
$$

The real sign integral TSMC should be:

$$
\bar{u}=\bar{u}_{e q}+\bar{u}_{s}
$$

where $e=y-y_{r}, \quad s=e+\lambda e_{I}, \quad \dot{e}_{I}=\operatorname{sgn}(e) \quad e_{I}(0)=-e(0) / \lambda$. Substitute (29) into (1):

$$
\dot{e}=-\lambda \operatorname{sgn}(e)
$$

It is obvious that $e$ and $\dot{e}$ converge to zero in finite time.

After $x_{2}$ converge to $x_{2 s}$, the concentration loop should be:

$$
\dot{x}_{1}=-x_{1}+D_{a}\left(1-x_{1}\right) e^{x_{2 s} /\left(x_{2 s} / \gamma+1\right)}-d_{1}
$$

Because $x_{2 s}$ is bounded $e^{x_{2 s} /\left(x_{2 s} / \gamma+1\right)}$ must be bounded. Note that $\left|d_{1}\right| \leq c_{1}$, hence $D_{a}\left(1-x_{1}\right) e^{x_{2 s} /\left(x_{2 s} / \gamma+1\right)}-d_{1}$ must be bounded. Let $\gamma_{1}=D_{a}\left(1-x_{1}\right) e^{x_{2 s} /\left(x_{2 s} / \gamma+1\right)}-d_{1}$, then (32) can be written as:

$$
\dot{x}_{1}=-x_{1}+\gamma_{1}
$$

Solving the ordinary differential equation (33), gives:

$$
x_{1}(t)=\left(x_{1}(0)-\gamma_{1}\right) e^{-t}+\gamma_{1}
$$

where $x_{1}(0)$ is the initial value of $x_{1}(t)$. It is obvious that $\lim _{t \rightarrow \infty} x_{1}(t)=\gamma_{1}$. Hence, $x_{1}(t)$ is asymptotically stable.

\section{B. Fraction integral terminal sliding mode control}

For (18), the estimated fraction integral terminal sliding mode is:

$$
\begin{aligned}
& \hat{s}=\hat{e}+\lambda \hat{e}_{I} \\
& \dot{\hat{e}}_{I}=|\hat{e}|^{q / p} \operatorname{sgn}(\hat{e}) \quad \hat{e}_{I}(0)=-\hat{e}(0) / \lambda
\end{aligned}
$$


The fraction integral terminal sliding mode control law can be designed as follows:

$$
\begin{gathered}
u_{e q}=\beta^{-1}\left(\hat{x}_{2}-B D_{a}\left(1-\hat{x}_{1}\right) e^{\hat{x}_{2} /\left(\hat{x}_{2} / \gamma+1\right)}+\beta\left(\hat{x}_{2}-x_{2 c}\right)\right. \\
\left.-d_{2}-\alpha_{2} \operatorname{sgn}\left(y-\hat{x}_{2}\right)+\dot{y}_{r}-\lambda|\hat{e}|^{q / p} \operatorname{sgn}(\hat{e})\right) \\
u_{s}=-K \operatorname{sgn}(\hat{s}) \\
u=u_{e q}+u_{s}
\end{gathered}
$$

where the control parameters are same as those of (24).

Theorem 3: Under Assumptions 1-3 and Properties 1-2, fraction integral TSMC (38) yields convergence of $\hat{e}$ and $\dot{\hat{e}}$ to 0 in finite time, implying $x_{2}$ will track to $y_{r}$ in finite time and $x_{1}$ is asymptotically stable.

Proof: The proof is similar to that of Theorem 2 and is omitted here.

Remark 4: It is important to find appropriate $\alpha_{1}$ and $\alpha_{2}$ in the controller design. In practice, trial and error method can be used to find these two parameters. First, a large enough $\alpha_{2}$ should be used to test the proposed control approach. Then, according to the control performance, one should increase/reduce $\alpha_{2}$ until an acceptable control performance is obtained. If $\alpha_{2}$ is well designed, $\alpha_{1}$ can be designed by using the same method.

Remark 5: Because $x_{1}$ is not measurable, an external disturbance observer was designed in Ref. 7 to estimate $x_{1}$ online. Refs. [33] and [34] use uncertainty observers to estimate $x_{1}$. The proposed approach develops a robust finite time observer, which can estimate $x_{1}$ online directly. The proposed observer is simpler and more effective than the existing approaches ${ }^{7,33-34}$. Compared with these existing sliding mode control algorithms of CSTR, the proposed control approach has a faster converging speed and stronger robustness, which is beneficial in industry

Remark 6: Note that if $q=p=1$, the fraction integral terminal sliding mode will be an asymptotical stable integral sliding mode control with a finite time stable observer, which is a special case of the proposed approach.

$$
\begin{gathered}
\hat{s}=\hat{e}+\lambda \hat{e}_{I} \\
\dot{\hat{e}}_{I}=\hat{e} \quad \hat{e}_{I}(0)=-\hat{e}(0) / \lambda \\
u_{e q}=\beta^{-1}\left(\hat{x}_{2}-B D_{a}\left(1-\hat{x}_{1}\right) e^{\hat{x}_{2} /\left(\hat{x}_{2} / \gamma+1\right)}+\beta\left(\hat{x}_{2}-x_{2 c}\right)\right. \\
\left.-d_{2}-\alpha_{2} \operatorname{sgn}\left(y-\hat{x}_{2}\right)+\dot{y}_{r}-\lambda \hat{e}\right)
\end{gathered}
$$




$$
\begin{gathered}
u_{s}=-K \operatorname{sgn}(\hat{s}) \\
u=u_{e q}+u_{s}
\end{gathered}
$$

\section{Simulation results}

A CSTR was investigated in this section, whose dynamics was described by (1). Its parameters were set as $B=8, \beta=0.3, \gamma=20, D_{a}=0.078, x_{2 c}=0$. The desired trajectory of system output was designed as:

$$
y_{r}=x_{2 s}\left(1-k_{1} e^{-k_{2} t}\right)
$$

where $x_{2 s}=2.7517, k_{1}=1, k_{2}=1$.

In this section, three types of control algorithms were tested in the absence of an external disturbance and in the presence of external disturbance, respectively. All the controllers' parameters were set to be the same to facilitate comparison. The controllers' parameters are listed in Table 2.

Table 2 The controllers' parameters

\begin{tabular}{|l|l|}
\hline \multicolumn{1}{|c|}{ Control algorithm } & \multicolumn{1}{|c|}{ Controller's parameters } \\
\hline Sign integral terminal sliding mode control (SITSMC) & $\begin{array}{l}\alpha_{1}=0.5, \alpha_{2}=0.5, K=0.2, \lambda=0.2, \\
\delta=0.05\end{array}$ \\
\hline Fraction integral terminal sliding mode control & $\begin{array}{l}\alpha_{1}=0.5, \alpha_{2}=0.5, K=0.2, \lambda=0.2, \\
\delta=0.05, q=7, \quad p=11\end{array}$ \\
\hline (FITSMC) & $\begin{array}{l}\alpha_{1}=0.5, \alpha_{2}=0.5, K=0.2, \lambda=0.2, \\
\text { Integral sliding mode control (ISMC) }\end{array}=0.05$ \\
\hline
\end{tabular}

Figure 2 shows the performance of SITSMC and FITSMC without using a smooth technique. In (a) and (d) $x_{1}$ and its estimation $\hat{x}_{1}$ are shown. In (b) and (e) $x_{2}$ and its estimation $\hat{x}_{2}$ are plotted. The control input is displayed in (c) and (f). From Figure 2, it can be seen that the estimated states converge to the real ones in the finite time. The system output $x_{2}$ can also converge to the desired trajectory $y_{r}$ in finite time. However, the control input is non-smooth as the sign control rather than the smooth control given by (39) is implemented. This phenomenon is called chattering. The chattering may wear and tear the actuators such as regulating valves. Figure 3 shows the performance of SITSMC, FITSMC and ISMC by using the smooth technique, in which the sign function is replaced by $z /(|z|+\delta), \delta>0$ is a small positive number. Comparing Figure 2 and 3, one can see 
the performances of these two approaches are very similar. Figure 3 shows that the chattering is eliminated effectively by using the smooth technique. Note that the performances of these approaches are very similar. This is because there are no external disturbances. If there are some disturbances, the control performances of these approaches will be very different. To further test the proposed approaches, external disturbance were added to the CSTR. They are step signals, $d_{1}=0.1, d_{2}=0.1$. Figure 4 shows the tracking performances of system output. In (a) $\hat{x}_{2}, x_{2}$ and $y_{r}$ of the SITSMC with using the smooth technique are shown. In (b) $\hat{x}_{2}, x_{2}$ and $y_{r}$ of the FITSMC with using smooth technique are presented and in (c) $\hat{x}_{2}, x_{2}$ and $y_{r}$ of the ISMC with a smooth technique are shown. It is obvious from Figure 4 that SITSMC gives the best results and ISMC gives the worst results.
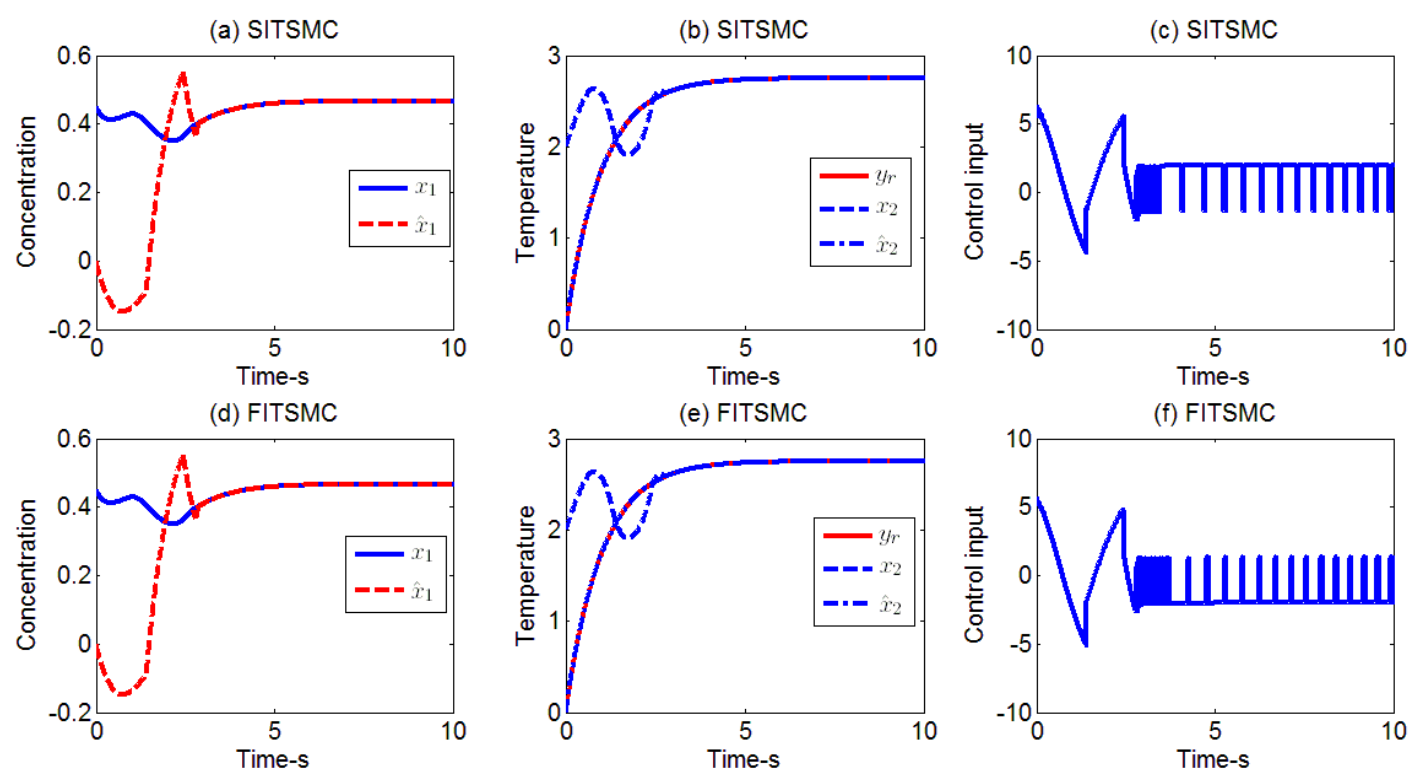

Figure 2 The performance of SITSMC and FITSMC (Non-smooth) 
(a) SITSMC

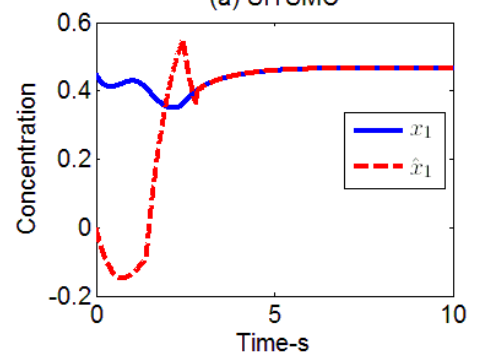

(d) FITSMC

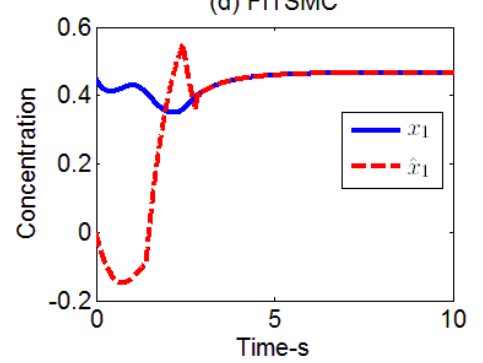

(b) SITSMC

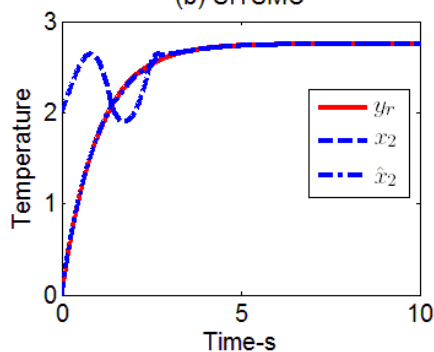

(e) FITSMC

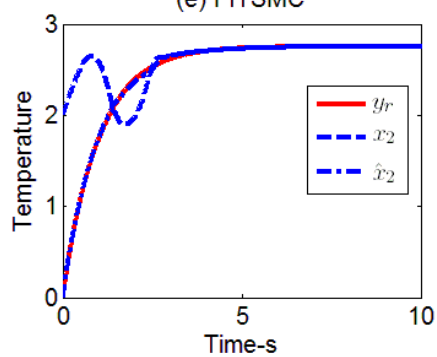

(c) SITSMC

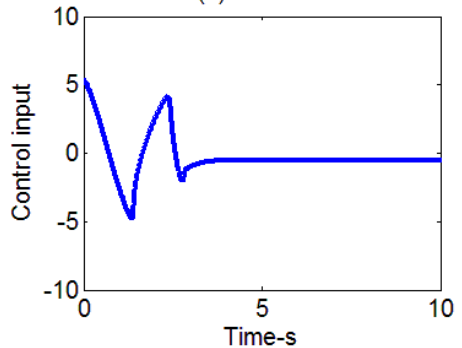

(f) FITSMC

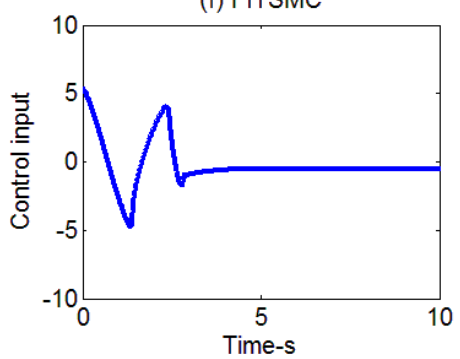

Figure 3 The performance of SITSMC, FITSMC (Smooth)

(a) SITSMC

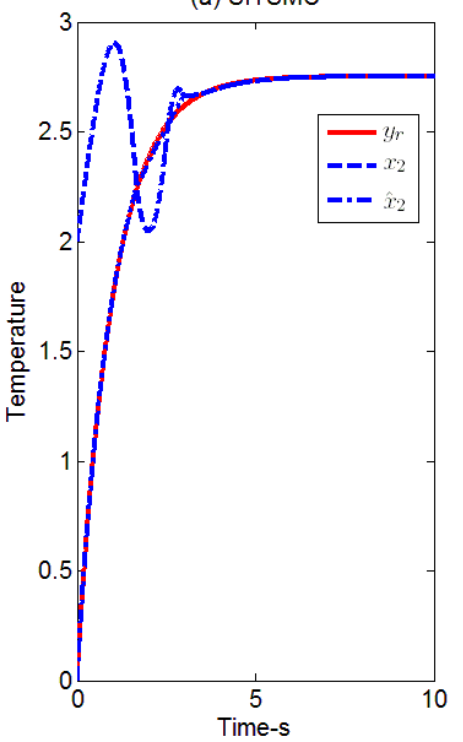

(b) FITSMC

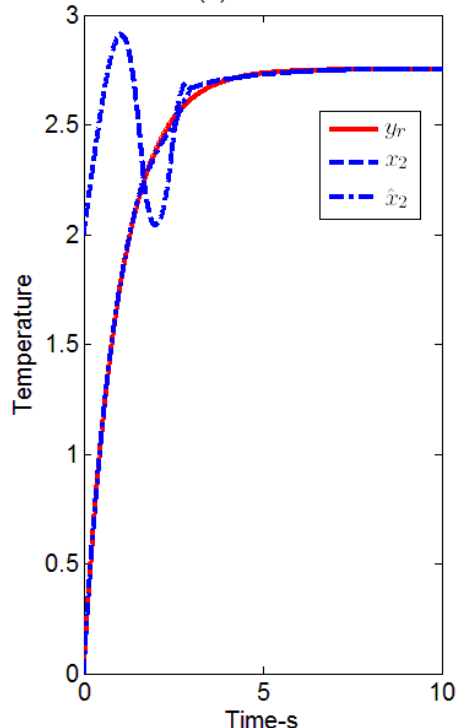

(c) ISMC

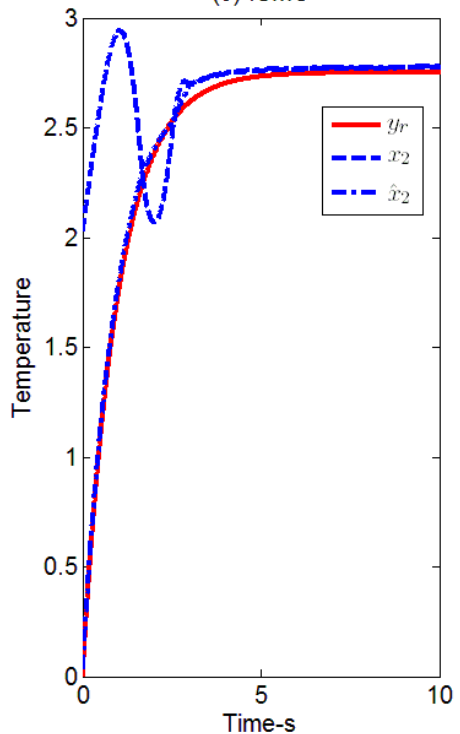

Figure 4 The performance of SITSMC, FITSMC and ISMC subjected to external disturbance (Smooth)

To show the performances of these three control approaches, time integrals multiplied by the error squared (ITSE) are given in Table 3. It illustrates again that the performance of SITSMC is the best. Although the smooth technique may degrade the control performance and lead to practical stability, the performances of the proposed controls are still acceptable for many practical purposes. This in contrast to the performance of the conventional sliding mode control which deteriorates heavily. The simulation results confirm the effectiveness of the proposed approaches. 
Table 3 Integral of time multiplied by squared error (ITSE)

\begin{tabular}{|l|l|}
\hline \multicolumn{1}{|c|}{ Control approach } & \multicolumn{1}{c|}{ ITSE } \\
\hline SITSMC (Non-smooth) & 1.0926 \\
\hline FITSMC (Non-smooth) & 1.0926 \\
\hline SITSMC (Smooth) & 1.1118 \\
\hline FITSMC (Smooth) & 1.1249 \\
\hline ISMC (Smooth) & 1.1318 \\
\hline SITSMC under external disturbance (Smooth) & 1.5782 \\
\hline FITSMC under external disturbance (Smooth) & 1.6366 \\
\hline ISMC under external disturbance f(Smooth) & 2.2128 \\
\hline
\end{tabular}

To further test the proposed approaches, they were compared with the ones of Ref. [33, 34]. Figure 5 shows the temperature track performances. From these results, one can see that the proposed approached is rapidly converging which is required in the practice.

(a) SITSMC

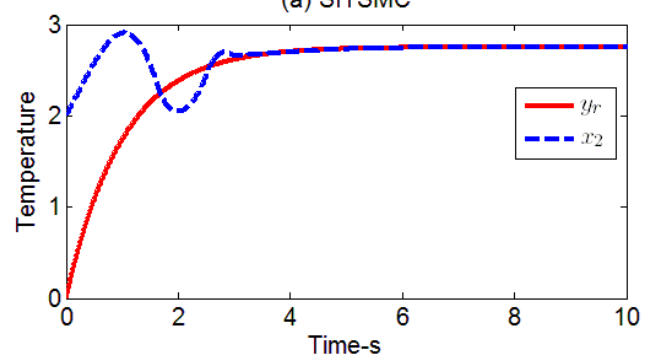

(c) Ref. [33]

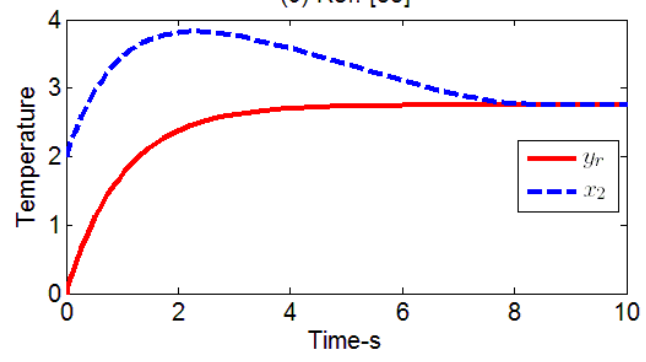

(b) FITSMC

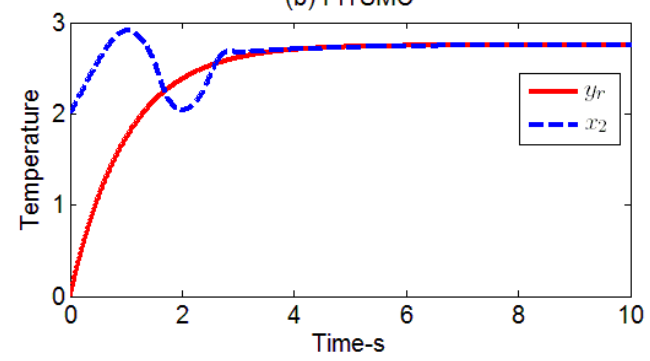

(d) Ref. [34]

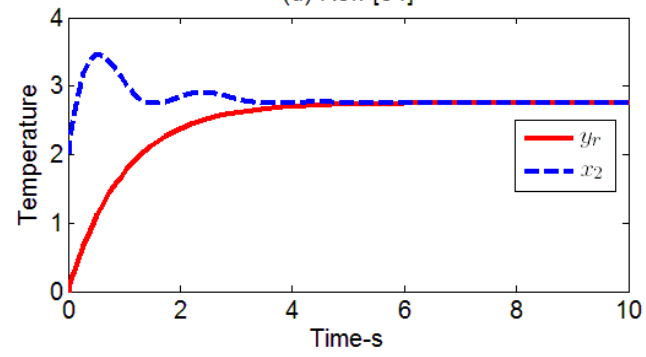

Figure 5 The comparison with Ref. [33, 34] 
(a) SITSMC

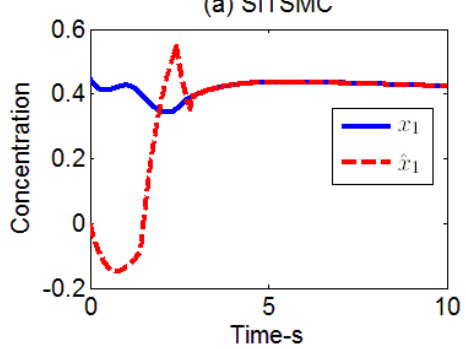

(d) FITSMC

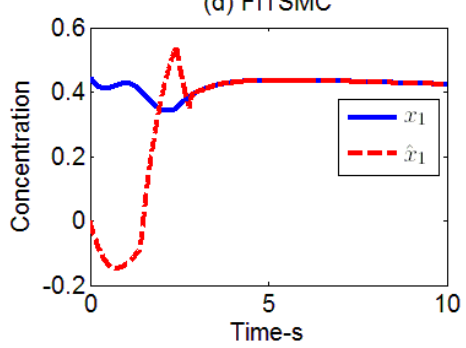

(b) SITSMC

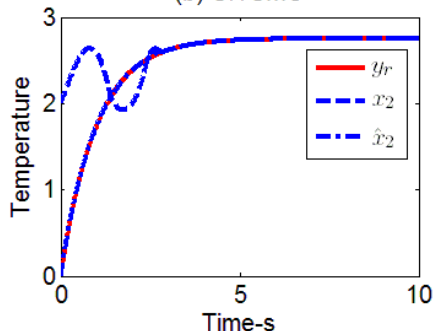

(e) FITSMC

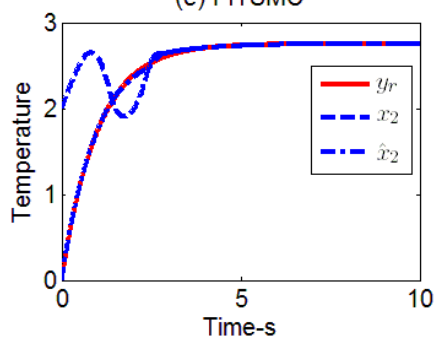

(c) SITSMC

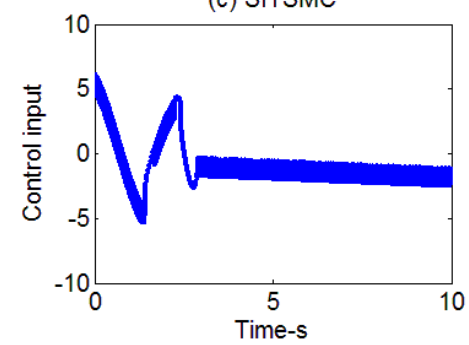

(f) FITSMC

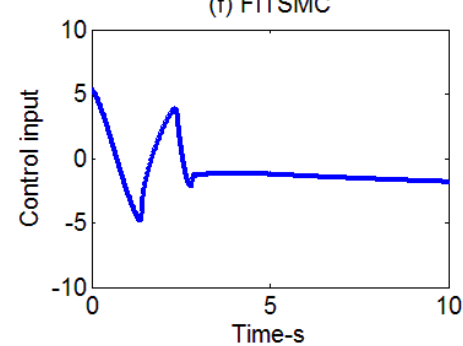

Figure 6 The performance of SITSMC, FITSMC under the sine disturbance (Smooth)

(a) SITSMC

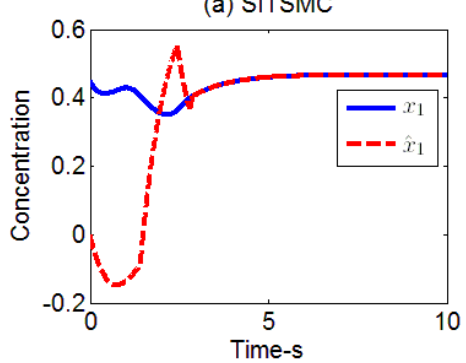

(d) FITSMC

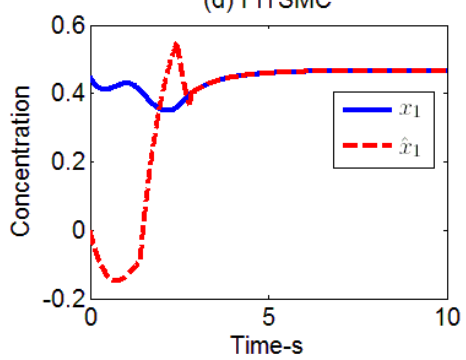

(b) SITSMC

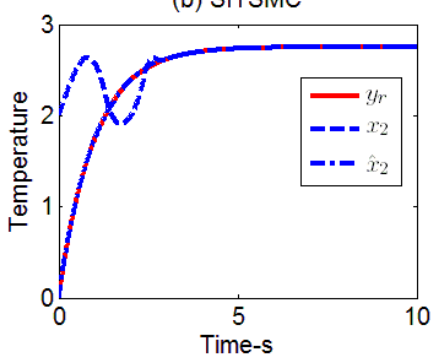

(e) FITSMC

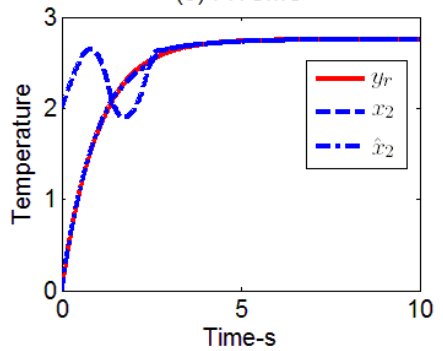

(c) SITSMC

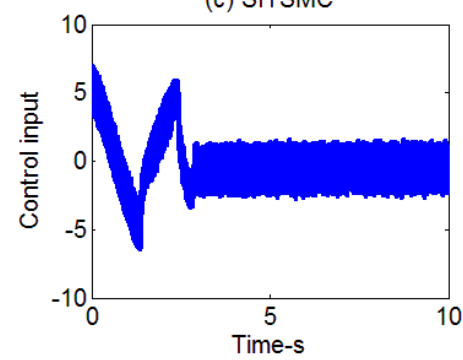

(f) FITSMC

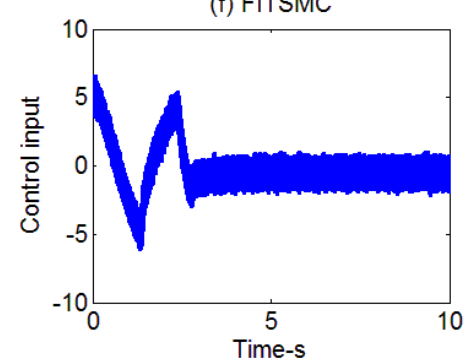

Figure 7 The performance of SITSMC, FITSMC under the Gaussian noise (Smooth)

To test the performance under rapid fluctuation disturbances, sine disturbance $d_{1}(t)=0.1 \sin (0.1 t)$ and $d_{2}(t)=0.1 \sin (0.1 t)$ are added to the system. Figure 6 shows the control performance. Gaussian noise disturbances with the similar amplitude are also added to the control system. Figure 7 shows the control performance. It is obvious that the designed observers can filter the rapid fluctuation disturbance and the designed terminal sliding mode controllers can obtain the acceptable control performance. Though the smooth technique is used in the controller design the control inputs are unsmooth due to the fluctuation disturbance. 


\section{Conclusions}

In this paper, TSMC approaches are initially developed for CSTR. By using sliding mode principles, a finite time stability observer is first designed to estimate the un-measurable states. Then, two novel output integral TSMC approaches, that is, SITSMC and FITSMC are developed for CSTR. Compared with existing linear integral sliding mode control, the proposed approaches have stronger robustness to external disturbances and can drive the tracking error to zero with faster converging speed. The corresponding stability analysis is presented to lay a theoretical foundation and a safe operation reference for potential applications. The effectiveness of the proposed approaches is validated through detailed numerical simulations. The presented approaches provide a more effective solution to CSTR. They have potential applications to other nonlinear process with relative degree one and stable zero dynamics. The immediate future work will be applying these new schemes to real CSTR experiments.

\section{Acknowledgements}

This work is partially supported by the National Nature Science Foundation of China under Grant 61004080, 61273188, Shandong Provincial Natural Science Foundation under Grant ZR2011FM003, China and the Fundamental Research Funds for the Central Universities of China, Postdoctoral Researcher Applied Research Project of Qingdao, Taishan Scholar Construction Engineering Special funding.

\section{Reference}

(1) Bequette, B. W. Process control: Modeling, Design, and Simulation, Prentice Hall: New Jersey, 2003.

(2) Saravanathamizhan, R.; Paranthaman, R.; Balasubramanian, N. Tanks in series model for continuous stirred tank electrochemical reactor. Industrial \& Engineering Chemistry Research, 2008, 47(9): 2976-2984.

(3) Ghaffari, V.; Naghavi, S. V.; Safavi, A. A. Robust model predictive control of a class of uncertain nonlinear systems with application to typical CSTR problems. Journal of Process Control, 2013, 23(4): 493-499.

(4) Flores-Tlacuahuac, A.; Grossmann, I. E. Simultaneous cyclic scheduling and control of a multiproduct CSTR. Industrial \& engineering chemistry research, 2006, 45(20): 6698-6712. 
(5) Cebuhar, W. A.; Costanza, V.: Nonlinear control of CSTR's. Chemical engineering science, 1984, 39(12): $1715-1722$

(6) Alvarez, J.; Alvarez, J,; González, E. Global nonlinear control of a continuous stirred tank reactor. Chemical engineering science, 1989, 44(5): 1147-1160.

(7) Colantonio, M. C.; Desages, A. C.; Romagnoli, J. A.; Palazoglu, A. Nonlinear control of a CSTR: disturbance rejection using sliding mode control. Industrial \& engineering chemistry research, 1995, 34(7): 2383-2392.

(8) Seki, H.; Naka, Y. Optimizing control of CSTR/distillation column processes with one material recycle. Industrial \& Engineering Chemistry Research, 2008, 47(22): 8741-8753.

(9) Kravaris, C.; Palanki, S. Robust nonlinear state feedback under structured uncertainty. AIChE Journal, 1988, 34(7): 1119-1127.

(10) Morari, M.; Zafiriou, E. Robust Process Control, Prentice Hall: New Jersey, 1989.

(11) Alvarez-Ramírez, J. Stability of a class of uncertain continuous stirred chemical reactors with a nonlinear feedback. Chemical engineering science, 1994, 49(11): 1743-1748.

(12) Kravaris, C.; Kantor, J. C. Geometric methods for nonlinear process control. 1. Background. Industrial \& Engineering Chemistry Research, 1990, 29(12): 2295-2310.

(13) Kravaris, C.; Kantor, J. C. Geometric methods for nonlinear process control. 2. Controller synthesis. Industrial \& Engineering Chemistry Research, 1990, 29(12): 2310-2323.

(14) $\mathrm{Wu}, \mathrm{W}$. Nonlinear bounded control of a nonisothermal CSTR. Industrial \& engineering chemistry research, 2000, 39(10): 3789-3798.

(15) Chen, C. T.; Peng, S. T. A nonlinear control scheme for imprecisely known processes using the sliding mode and neural fuzzy techniques. Journal of Process Control, 2004, 14(5): 501-515.

(16) Pan, T.; Li, S.; Cai, W. J. Lazy learning-based online identification and adaptive PID control: a case study for CSTR process. Industrial \& engineering chemistry research, 2007, 46(2): 472-480.

(17) Graichen, K.; Hagenmeyer, V.; Zeitz, M. Design of adaptive feedforward control under input constraints for a benchmark CSTR based on a BVP solver. Computers \& Chemical Engineering, 2009, 33(2): 473-483.

(18) Di, Ciccio, M. P.; Bottini, M.; Pepe, P.; Foscolo, P. U. Observer-based nonlinear control law for a continuous stirred tank reactor with recycle. Chemical Engineering Science, 2011, 66(20): 4780-4797.

(19) Hoang, H.; Couenne, F.; Jallut, C.; Gorrec Y. L. Lyapunov-based control of non isothermal continuous stirred tank reactors using irreversible thermodynamics. Journal of Process Control, 2012, 22(2): 412-422.

(20) Antonelli, R.; Astolfi, A. Continuous stirred tank reactors: easy to stabilise? Automatica, 2003, 39(10): 
$1817-1827$.

(21) Zhao, D.; Li, S.; Zhu, Q.; Gao, F. Robust finite-time control approach for robotic manipulators. IET control theory \& applications, 2010, 4(1): 1-15.

(22) Zhao, D.; Li, S.; Zhu, Q. Output Feedback Terminal sliding mode control for a class of second order nonlinear systems. Asian Journal of Control, 2013, 15(1): 237-247.

(23) Chiu, C. S. Derivative and integral terminal sliding mode control for a class of MIMO nonlinear systems. Automatica, 2012, 48(2): 316-326.

(24) Yang, J.; Li, S.; Su. J.; Yu, X. Continuous nonsingular terminal sliding mode control for systems with mismatched disturbances. Automatica, 2013, 49(7): 2287-2291.

(25) Feng, Y.; Yu, X.; Han, F. On nonsingular terminal sliding-mode control of nonlinear systems. Automatica, 2013, 49(6): 1715-1722.

(26) Feng, Y.; Yu, X.; Han, F. High-order terminal sliding-mode observer for parameter estimation of a permanent magnet synchronous motor. IEEE Transactions on Industrial Electronics, 2013, 60(10): 4272-4280.

(27) Du, H.; Li, S. Finite-time cooperative attitude control of multiple spacecraft using terminal sliding mode control technique. International Journal of Modelling, Identification and Control, 2012, 16(4): 327-333.

(28) Zhao, D.; Li, S.; Gao, F.; Zhu, Q. Robust adaptive terminal sliding mode-based synchronised position control for multiple motion axes systems. IET Control Theory and Applications, 2009, 3(1): 136-150.

(29) Chen, S.-Y.; Lin, F.-J. Robust nonsingular terminal sliding-mode control for nonlinear magnetic bearing system. IEEE Transactions on Control systems technology, 2011, 19(3): 636-643.

(30) Almutairi, N. B.; Zribi, M. Control of a Plate Heat Exchanger Using the Terminal Sliding Mode Technique. Industrial \& Engineering Chemistry Research, 2012, 51(12): 4610-4623.

(31) Yan, X.-G.; Spurgeon, S. K.; Edwards, C. Sliding mode control for time-varying delayed systems based on a reduced-order observer. Automatica, 2010, 46(10): 1354-1362.

(32) Yan, X.-G.; Spurgeon, S. K.; Edwards, C. State and parameter estimation for nonlinear delay systems using slding mode techniques. IEEE Transactions on Automatic Control, 2013, 58(4): 1023-1029.

(33) Aguilar-López, R.; Alvarez-Ramírez, J. Sliding-mode control scheme for a class of continuous chemical reactors. IEE Proceedings-Control Theory and Applications, 2002, 149(4): 263-268.

(34) Chen, C. T.; Peng, S. T. A sliding mode control scheme for uncertain non-minimum phase CSTRs. Journal of chemical engineering of Japan, 2006, 39(2): 181-196.

(35) Daly, J. M.; Wang, D. W. L. Output feedback sliding mode control in the presence of unknown disturbance. 
Systems \& Control Letters, 2009, 58(3): 188-193.

(36) Slotine, J. J. E.; Li, W. Applied nonlinear control. Prentice hall: New Jersey, 1991.

(37) Utkin, V.; Guldner, J.; Shi, J. Sliding Mode Control in Electro-Mechanical Systems. CRC Press: New York, 2009. 